Sharif University of Technology
Scientia Iranica
SCIENTIA
I RAN I CA

\title{
Arc based ant colony optimization algorithm for solving sewer network design optimization problem
}

\author{
R. Moeini* \\ Department of Civil Engineering, University of Isfahan, Postal Code: 81746-73441, Isfahan, Iran.
}

Received 31 August 2015; received in revised form 30 January 2016; accepted 10 May 2016

KEYWORDS
Arc based ant
colony optimization
algorithm;
Sewer network;
Exploration;
Exploitation;
Heuristic information;
Optimal design.

\section{Introduction}

Nowadays, optimization is one of the most important fields of any engineering problems. In optimization problems, one should find the minimum/maximum of a function of many variables, called decision variables, where the arguments may be subjected to some constraints. Cost saving is a major goal of most engineering problems, such as design and implementation of a sewer network. Due to the high cost associated with the construction of a sewer network, many research studies over the last 50 years have attempted to develop different techniques in which they are used to minimize the capital costs associated with such infrastructure. However, optimal design of a sewer

*. Tel.: 0098-31-37935293; Fax: 0098-31-37935231

E-mail address: r.moeini@eng.ui.ac.ir network is a highly constrained mixed-integer nonlinear programming (MINLP) problem presenting a challenge even to the modern heuristic search methods due to continues and discrete variables, nonlinear functions, and the high number of constraints involved [1].

Within the last decade, many researchers have focused on the sewer network design optimization problem and proposed different methods from traditional optimization techniques to modern heuristic search methods. The methods used for sewer network design optimization problem can be classified as: 1) Linear Programming (LP); 2) Nonlinear programming (NLP); 3) Dynamic Programming (DP); 4 ) meta-heuristic; and 5) hybrid methods. Each of these methods has its own limitations. It is worth noting that, over the last 30 years, a new kind of approximate method, called meta-heuristic, has been proposed that tries to combine basic heuristic methods in higher level 
structures aimed to explore a search space efficiently and effectively. This method is based on the fact that nature has the best built-in optimization techniques for maintaining the global systems. Sharing the information between insects, such as finding the shortest path of the food sources by ants, brings about the idea of optimization techniques that have opened a wide new field for research. This class of method includes, but is not restricted to, Genetic Algorithms (GA), Simulated Annealing (SA), Tabu Search (TS), Particle Swarm Optimization (PSO) algorithm, Ant Colony Optimization Algorithm (ACOA), etc. In the last three decades, the use of this method has extremely received the attention of the researchers in different engineering fields. However, the main limitation of this method is problem dependence. In other words, none of the proposed meta-heuristic methods is unique to all types of engineering problems. Also, the application of this method to real-world problem is full of complexities.

In the field of sewer network design optimization problem with fixed layout, many different research studies have been studied. Haestad [2] and Guo et al. [3] have reviewed the research studies of this field over the last 40 years. In general, traditional methods [4], LP [5-7], NLP [8,9], DP [10-16], metaheuristic [17-32], and hybrid methods [33-38] have been used in this field.

The ACOA is one of the meta-heuristic methods in which it mimics the natural foraging behavior of a colony of real ants to find the shortest path between the food source and their nest. When ants are traveling, they deposit a substance called pheromone forming a pheromone trail, which is more attractive to other ants to follow them. Ants can smell the pheromone and when choosing their path, they incline to choose, in all probability, paths marked by strong pheromone concentrations. It has been shown experimentally that this pheromone trail following behavior can give rise, once employed by a colony of ants, to the emergence of the shortest paths. This particular behavior of ant colonies has inspired the ACOA in which a colony of artificial ants cooperates to find good solutions for discrete optimization problems. The basic algorithm of ACOA is the Ant System (AS). Many other algorithms, such as Ant Colony System (ACS), elitist Ant System $\left(\mathrm{AS}_{\text {elite }}\right)$, elitist-rank Ant System $\left(\mathrm{AS}_{\text {rank }}\right)$, and MaxMin Ant System (MMAS), have been introduced to improve the performance of the AS.

Due to the iterative nature of the solution generations of meta-heuristic methods, the solution space uses the knowledge about solutions that have already been found to further guide the search. The searching behavior of the method can be described by two main features, named exploration and exploitation. It is worth noting that the exploration is the ability of the algorithm to search extensively through the solution space, and the exploitation is the ability of the method to search more comprehensively in the local neighborhood where good solutions have previously been found. However, these features are in conflict with each other. One of the most difficult aspects to pay attention to in meta-heuristic method is the tradeoff between these two features. To obtain good results, an agent should prefer actions tried in the past and found to be effective in producing proper solutions, but to discover them, he has to try actions not previously selected [39].

In this paper, a new formulation, i.e. Arc Based ACOA (ABACOA) formulation, is used to solve sewer network design optimization problem. The ABACOA was previously proposed for single and multi-reservoir operation problems by Moeini and Afshar $[39,40]$. The ABACOA has two significant advantages over the alternative point-based formulation as presented in the following. First, the pheromone trails are associated with the arcs of the graph leading to a more efficient implementation of the exploration and exploitation features of the ACOA. Second, each arc of the proposed graph is representative of downstream and upstream cover depths of each nodal pipe, and hence, average pipe cover depths lead to an easy and straightforward definition of the heuristic information for the ants, so there will be a useful property that is absent in the existing alternative formulations. It is worth noting that the performances and abilities of this new formulation, ABACOA, should be studied to solve different optimization problems, such as sewer network design optimization problem, considered here.

To show the unique feature of the proposed ABACOA, in this paper, this algorithm is used to solve sewer network design optimization problem by proposing two different formulations. In both formulations, the cover depths of sewer network nodes are taken as decision variables of the problem. In the first formulation, named UABAC, the unconstrained version of $\mathrm{ABACOA}$ is used to determine the cover depths of sewer network nodes. However, in the second formulation, named CABAC, the characteristics of the arc based definition of the problem along with the serial features of sewer network problem are then used to develop a constrained version of the formulation. The constrained version of the ABACOA is proposed here for the explicit satisfaction of slope constraint. The constrained version is used to recognize the infeasible regions of the search space and remove them from the search space of the problem. Two benchmark test examples are solved here using the proposed methods, and the results are presented and compared with unconstrained and constrained versions of point-based ACOA (PBACOA) and with those obtained by the other existing methods. 


\section{Sewer network design optimization model}

Sewer network is one of the most important infrastructures of modern city which is designed to collect sewerages from city and transfer them to wastewater treatment plants. A sewer network consists of manholes, pipes, lifts and pumping stations, and other appurtenances. Sewer network is a more expensive infrastructure. Therefore, an optimization model should be defined to design the least-cost sewer network. The sewer network components can be optimally found by solving this optimization model [1].

The objective functions and constraints of the optimization model should be defined and be solved by effective methods in order to find sewer network parameters such as pipe diameters, slopes, average pipe cover depths, drops and pumping station locations and heights. The objective function of this optimization model is minimization of sewer network construction cost. In the absence of any pumps and drops, it can be formulated for gravitational sewer network as follows [37]:

$$
\text { Minimize } C=\sum_{l=1}^{N P} L_{l} K_{p i p}\left(d_{l}, E_{l}\right)+\sum_{n m=1}^{N M} K_{m a n}\left(h_{n m}\right),
$$

where:

$\begin{array}{ll}C & \begin{array}{l}\text { Construction cost function of sewer } \\ \text { network }\end{array} \\ N P & \text { Total number of sewer pipes } \\ N M & \text { Total number of manholes } \\ L_{l} & \text { Length of pipe } l(l=1, \ldots, N P) \\ K_{\text {pip }} & \text { The unit cost of sewer pipe provision } \\ & \text { and installation defined as a function } \\ & \text { of its diameter }\left(d_{l}\right) \text { and average cover } \\ & \text { depth }\left(E_{l}\right) \\ & \text { The construction cost of manhole as a } \\ K_{\text {man }} & \text { function of manhole height }\left(h_{n m}\right)\end{array}$

Furthermore, the constraints of the sewer network design optimization model with a pre-specified layout are hydraulic, operational, and availability constraints which are formulated as:

$$
\begin{array}{ll}
V_{\min } \leq V_{l} \leq V_{\max } & \forall l=1, \ldots, N P, \\
S_{l} \geq S_{\min } & \forall l=1, \ldots, N P, \\
E_{\min } \leq E_{l} \leq E_{\max } & \forall l=1, \ldots, N P, \\
\beta_{\min } \leq \beta_{l} \leq \beta_{\max } & \forall l=1, \ldots, N P, \\
\beta_{l}=\left(\frac{y}{d}\right)_{l} & \forall l=1, \ldots, N P, \\
Q_{l}=\frac{1}{n} a_{l} r_{l}^{2 / 3} S_{l}^{1 / 2} & \forall l=1, \ldots, N P,
\end{array}
$$

$$
\begin{array}{ll}
d_{l} \in \mathbf{D} & \forall l=1, \ldots, N P, \\
d_{l} \leq \mathbf{d}_{l} & \forall l=1, \ldots, N P,
\end{array}
$$

where:

$V_{l} \quad$ Flow velocity of pipe $l$ at the design flow

$V_{\max } \quad$ Maximum allowable velocity of sewer flow

$V_{\min } \quad$ Minimum allowable velocity of sewer flow

$S_{l} \quad$ Slope of the sewer pipe

$S_{\min } \quad$ Minimum sewer pipe slope

$E_{\text {min }} \quad$ Minimum cover depth of sewer pipe

$E_{\max } \quad$ Maximum cover depth of sewer pipe

$E_{l} \quad$ Average cover depth of pipe $l$

$d_{l} \quad$ Diameter of sewer pipe $l$

$y_{l} \quad$ Sewer flow depth in pipe $l$

$\beta_{\max } \quad$ Maximum allowable relative flow depth

$\beta_{\text {min }} \quad$ Minimum allowable relative flow depth

$\beta_{l} \quad$ Relative flow depth of pipe $l$

$Q_{l} \quad$ The discharge of sewer pipe $l$

$a_{l} \quad$ Wetted cross-section area of sewer pipe $l$ at sewer flow depth of $y_{l}$

$r_{l} \quad$ Hydraulic radius of the sewer pipe $l$ at sewer flow depth of $y_{l}$

n Manning coefficient

D Discrete set of commercially available sewer pipe diameters assumed equal for all pipes of the network

$\mathbf{d}_{l} \quad$ Set of downstream pipe diameters of pipe $l$

This optimization model of the sewer network design with a pre-specified layout (Eqs. (1) to (9)) is a highly constrained mixed-integer nonlinear problem (MINLP) in which the complexity of the problem requires that an effective method be proposed to solve it.

\section{The proposed methods for solving optimization model}

Herein, ACOA is used to solve sewer network design optimization problem. The basic steps of the ACOA for solving optimization problem were presented by Afshar and Moeini [41], and therefore, will not be presented here. However, Figure 1 shows the basic steps of ACOA procedure briefly to solve optimization problem. Formulation of an optimization problem by ACOA requires that the problem be defined as graph $\mathbf{G}=\left(\mathbf{D P}, \mathbf{O P}_{\mathbf{i}}, \mathbf{C O}\right)$. This graph is defined by a set of nodes referred to as decision points, $\mathbf{D P}=$ 


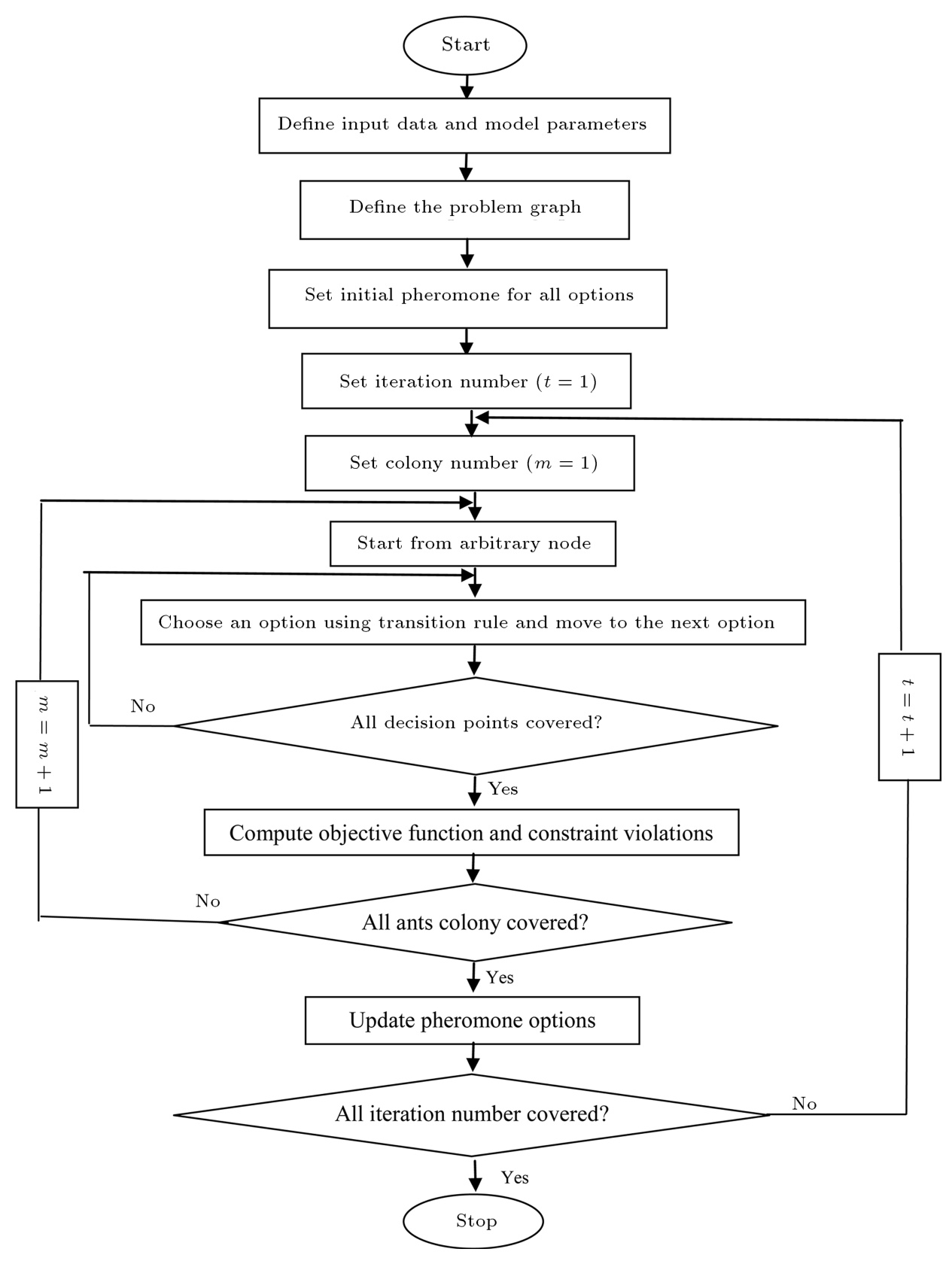

Figure 1. ACOA procedure to solve optimization problem.

$\left\{d p_{1}, d p_{2}, \ldots, d p_{I}\right\}$, and edges referred to as options available at each decision point $i, \mathbf{O P}_{\mathbf{i}}=\left\{o p_{i j}\right\}$ with corresponding costs $\mathbf{C O}=\left\{c o_{i j}\right\},[1]$.

Here, an arc based formulation of ACOA is proposed by defining another form of the graph for its efficient application to solve sewer network design optimization problem. This formulation has two significant advantages over the alternative usual point based formulation. The significance of the proposed formulation is that each arc of the new defined graph is representative of some parameters leading to an easy and straightforward definition of the heuristic information for the ants, which is a useful property absent in the existing alternative point-based formula- tion. Furthermore, the pheromone trails are associated with the arcs of the new defined graph leading to more efficient implementation of the exploration and exploitation features of the ACOA. The characteristics of the arc based ACOA along with the serial feature of sewer network optimization problem are also used to develop a constrained version of arc based ACOA. Furthermore, usual form of the graph for ACOA is also used to propose unconstrained and constrained pointbased ACOA formulations, i.e. UPBAC and CPBAC, respectively, for comparison purposes. It should be noted that these formulations are the modified forms of Afshar's [21] formulations with minor corrections.

It is worth noting that the problem graph is 
very much dependent on the decision variables of the problem. Here, in the absence of pumps and drops, the cover depths of the sewer network nodes are considered as the decision variables of the problem leading to an easy definition of the problem graph. Furthermore, an assumption used here is that the gravitational sewer network is considered. This assumption leads to the fact that, for each node, the downstream nodal elevations of entering pipes are the same as upstream nodal elevations of outgoing pipe.

It should be noted that the proposed formulations are used here for sewer network with predefined layout, and therefore, the sewer discharge is known. In other words, the steady-state condition is considered for hydraulic model. Furthermore, the proposed formulations are outlined here using MMAS for sewer network design optimization problem.

\subsection{Unconstrained Point-Based ACOA ( UPBAC)}

In this formulation, named UPBAC, the conventional application of ACOA based on the usual form of the graph is used to determine decision variables. By considering the sewer network nodes as decision points, the options available at each decision point are represented by all finite numbers of discrete cover depths of sewer network nodes. The graph representation of this formulation is shown in Figure 2, where vertical lines represent the decision points (nodes), circles represent the components of nodal cover depths $(j=1, \ldots, J)$ at each decision point $i(i=1, \ldots, I)$, the dash lines represent potential solutions on the graph, bold circles represent nodal cover depths selected by arbitrary ant, and finally, the bold lines represent a trial solution on the graph constructed by an arbitrary ant. In this formulation, each ant starts its movement from an arbitrary decision point and selects a cover depth from the set of available cover depths for each node. It should be noted that based on the methodology of UPBAC formulation, the construction of layout with negative slope is possible in which it is an infeasible solution. Here, penalty method is used to avoid infeasible solution, which is explained later.

By determining the nodal cover depths, nodal elevations and pipe slopes of sewer network, the sewer

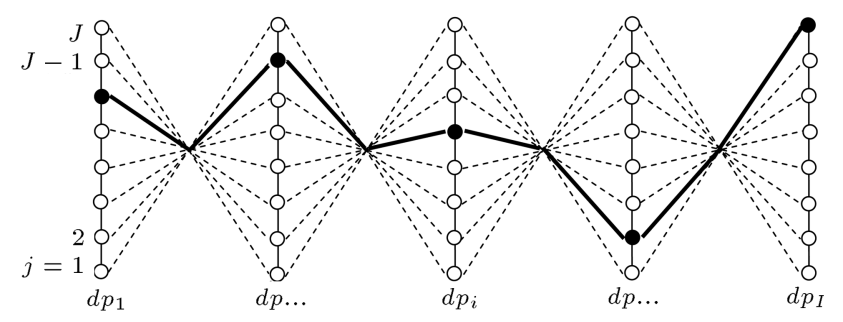

Figure 2. Problem graph of unconstrained version of point based ACOA. network pipes' diameters should be determined to complete the design process. Different methods can be used to calculate the pipe diameters when the pipe slopes are known at steady-state condition. Here, the pipe diameters are calculated explicitly, such that all the constraints are fully satisfied, if possible. Therefore, by starting the design processes from upstream pipes, the smallest commercially available diameter fully satisfying Constraints (2), (5), (6), (8), and (9) is taken as sewer pipe diameter.

\subsection{Constrained Point-Based ACOA $(C P B A C)$}

In CPBAC formulation, the constrained version of ACOA based on the usual form of the graph is used to determine decision variables. The constrained version of point-based ACOA is proposed for the explicit enforcement of the problem constraints by limiting the ant's options to feasible ones at each decision point of the problem. This leads to limiting the search space of the problem to feasible region, and therefore will be shown to improve convergence characteristics and obtain better results.

By considering the sewer network nodes as decision points, in this formulation, the options available at each decision point are represented by a finite number of discrete cover depths of sewer network nodes, which have satisfied minimum slope constraint. Here, for a sewer network with predefined layout, each ant starts its movement form inlets and selects an option, cover depth, from the available feasible options' list. In other words, the feasible cover depths of downstream nodes can be easily defined using known upstream cover depths and minimum slope constraint. Figure 3 represents the graph of this formulation, where vertical lines represent the decision points (sewer network nodes), circles represent the components of nodal cover depths $(j=1, \ldots, J)$ at each decision point $i(i=1, \ldots, I)$ with the dashed ones representing the infeasible and the solid one representing feasible cover depths, the dash lines represent potential solutions on the graph, bold circles represent nodal cover depths selected by arbitrary ant, and finally, the bold lines represent a trial solution on the graph constructed by an arbitrary ant. It is worth noting that, in contrast to UPBAC formulation, in this formulation, each ant starts its

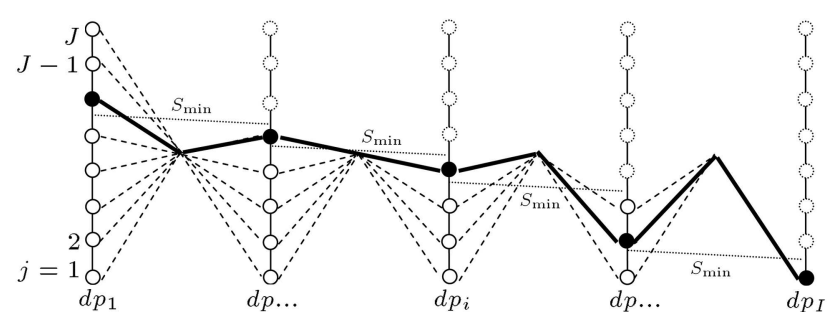

Figure 3. Problem graph of constrained version of point based ACOA. 
movement from inlets of sewer network and selects a cover depth from feasible cover depths' list, which is much smaller than original list. By determining the nodal cover depths, other parameters are determined such as UPBAC formulation.

\subsection{Unconstrained Arc Based ACOA ( $U A B A C$ )}

In UABAC formulation, the arc based ACOA is used to determine decision variables. With the nodal cover depths taken as the decision variables, in this formulation, the allowable range of the nodal cover depths is discretised into a fixed number of $J$ discrete values, and each discrete point $j(j=1,2, \ldots, J)$ is taken as the decision point of the problem. The set of options, $O P_{i}$, available at a decision point $i$ will then be represented by a total number of $J$ arcs joining the decision point, $i$, representing the $j$ th discrete upstream nodal cover depth to all discrete downstream nodal cover depths of corresponding pipe. Figure 4 represents the graph of this formulation, where vertical lines represent the sewer network nodes, circles represent the components of discretized nodal cover depths $(j=1, . ., J)$ representing decision points $d p_{i}$ $(i=1, \ldots, I)$, the dash lines represent arcs connecting two decision points, bold circles represent nodal cover depths selected by arbitrary ant, and finally, the bold lines represent a trial solution on the graph constructed by an arbitrary ant.

It is worth noting that, in this formulation, each ant starts its movement from inlets and it only has to choose one arc out of the total number of $J$ arcs joining the decision point under consideration to the decision points of the corresponding pipe which represent upstream and downstream nodal elevations of each pipe, respectively. Once an option, an arc, is chosen by an ant, the next decision point to move to is known. This process continues when all decision points of the problem are covered by ant. Finally, by determining the nodal cover depths, other parameters are determined such as UPBAC formulation. It should be noted that based on the methodology of this formulation, the construction of layout with negative slope is possible in which it is an infeasible solution.

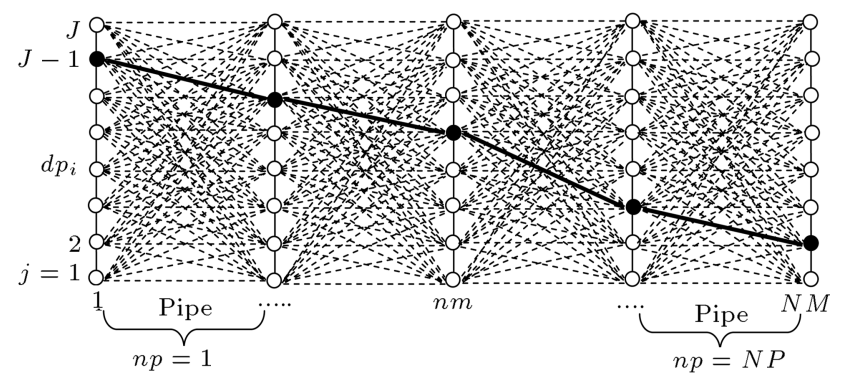

Figure 4. Problem graph of unconstrained version of arc based ACOA.
Here, penalty method is also used to avoid infeasible solution, which is explained later.

The arc based formulation has significant advantages over the alternative point-based formulation, which are presented before. Heuristic information, $\eta_{i j}$, is a useful component of the ACOA in which the proper definition of it is required for the best algorithm performance. Generally, heuristic value represents the costs of choosing option $j$ at point $i$, and therefore, related to the cost function equation. Here, based on the sewer network cost function of Eq. (1), the cost function of sewer network is only related to the average cover depths and diameters of sewer pipe, and therefore, the heuristic information can be easily computed in the proposed arc based formulation. As noted earlier, in the proposed arc based formulation, each arc will uniquely define the value of the upstream and downstream nodal cover depths of each pipe. This in turn leads to the possibility of defining average cover depth of each pipe, $E_{l}$, leading to the possibility of defining heuristic information. In other words, this in turn leads to the possibility of defining some part of cost function, and consequently heuristic information for each arc. This possibility does not exist in the alternative usual point-based formulation in which each option only defines the value of the cover depths of corresponding nodes with which no cost can be associated. The heuristic information of the problem can, therefore, be defined as:

$$
\begin{aligned}
& \eta_{i j}=\frac{1}{k_{p i p}\left(E_{l}\right)}, \\
& E_{l}=\frac{E_{i}+E_{j}}{2},
\end{aligned}
$$

where all parameters have been defined before. Furthermore, in the proposed arc based formulation, the pheromone trails are now associated with the arcs of the graph leading to more efficient implementation of the exploration and exploitation mechanisms of the ACOAs. These points will be verified later when considering numerical examples.

\subsection{Constrained Arc Based ACOA (CABAC)}

In this formulation, named $\mathrm{CABAC}$, the proposed arc based ACOA formulation is augmented with the interracial building capability of ACOA by proposing the constrained version of arc based ACOA formulation. The constrained version of arc based ACOA is proposed for the explicit enforcement of the minimum slope constraint by limiting the ant's options to feasible ones at each decision point of the problem.

With the nodal cover depths also taken as the decision variables, in this formulation, the allowable range of the nodal cover depths is discretized into a fixed number of $J$ discrete value, and each discrete 


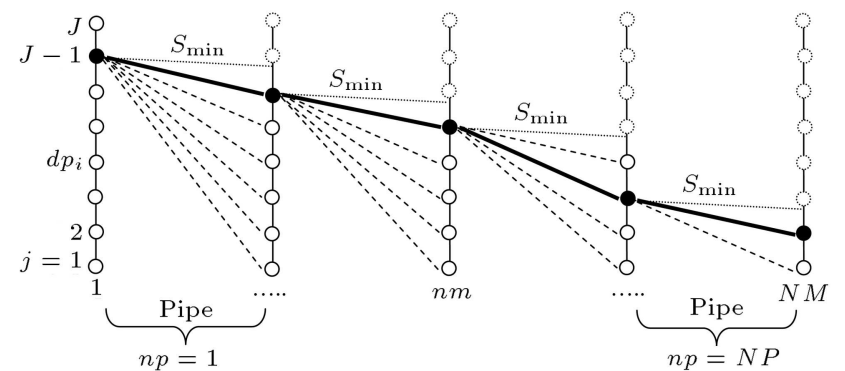

Figure 5. Problem graph of constrained version of arc based ACOA.

point $j(j=1,2, \ldots, J)$ is taken as the decision point of the problem. The set of options, $O P_{i}$, available at a decision point $i$, will then be represented by a number of $J$ arcs joining the decision point $i$, representing the $j$ th discrete upstream nodal cover depth, to some discrete downstream nodal cover depths of the corresponding pipe satisfying minimum slope constraint. In other words, in the proposed CABAC, the ant is forced to choose an option only from those arcs which have satisfied minimum slope constraint. The modified graph representation of the problem for the application of the proposed CABAC is shown in Figure 5, in which, circles represent the components of discretized nodal cover depths $(j=1, \ldots, J)$ representing decision points, $d p_{i}(i=1, \ldots, I)$, in which the dash circle represents the infeasible ones; the dash lines represent arcs connecting two decision points satisfying minimum pipe slope constraint; bold circles represent nodal cover depths selected by an arbitrary ant; finally, the bold lines represent a trial solution on the graph constructed by an arbitrary ant. It can be clearly seen from Figure 5 that the resulting search space is much smaller than the original search space, as shown in Figure 4. Therefore, it is expected that the resulting formulation will perform better than the UABAC.

It is worth noting that the proposed formulations, UPBAC, CPBAC, UABAC, and CABAC, however, may lead to trial solutions that may violate some of the problem constraints. To discourage the ants to make decisions which constitute an infeasible solution, higher costs are associated with the solutions that violate the problem constraints. This is achieved via the use of a penalty method in which the total cost of the problems is considered as the sum of the problems cost and penalty cost as follows:

$$
C_{p}=C+\alpha_{p} \times \sum_{k=1}^{K} C S V_{k}
$$

where $C$ is the cost function associated with the objective functions (Eq. (1)); $C_{p}$ is the penalized cost function; $C S V_{k}$ is the value of the $k$ th constraint violation; and $\alpha_{p}$ represents the penalty parameter.

\section{Test examples, results, and discussions}

In this section, the performance of the proposed formulations is tested against two benchmark examples from the literature. The first one (test example I) is the case of a network originally proposed by Mays and Yen [42], then considered again by Mays and Wenzel [43], and also solved by other researchers. This sewer network has 21 nodes and 20 pipes (Figure 6). This network is constrained to a maximum flow velocity of $12 \mathrm{fps}$, minimum flow velocity of $2 \mathrm{fps}$, maximum relative flow depth of 0.9 , minimum relative flow depth of 0.1 , and minimum cover depth of $8 \mathrm{ft}$. It is assumed here that the pipes have the variable Manning coefficient with the value of 0.013 at full condition. Details of the network, including nodal ground elevations and length and design discharge of each pipe, are given in Table 1. Commercial pipe diameters are 12, 15, 18, 21, 24, 30, 36,42 , and 48 inches, and other problem data of this test example are presented in the paper of Mays and Wenzel [43]. Here, the following relation is used for pipe installation and manhole costs:

$$
\begin{gathered}
K_{\text {pip }}=\left\{\begin{array}{c}
10.98 d_{l}+0.8 E_{l}-5.98 \\
\text { if } d_{l} \leq 3^{\prime} \text { and } E_{l} \leq 10^{\prime} \\
5.94 d_{l}+1.166 E_{l}+0.504 d_{l} E_{l}-9.64 \\
\text { if } d_{l} \leq 3^{\prime} \text { and } E_{l} \geq 10^{\prime} \\
30.0 d_{l}+4.9 E_{l}-105.9 \\
\text { if } d_{l}>3^{\prime}
\end{array}\right. \\
K_{\text {man }}=250+h_{m}^{2} .
\end{gathered}
$$

The second one (test example II) is the case of a part of the network designed by Mansoury and Khanjani [44] for 'Kerman' city, Iran. This sewer network has 21 nodes and 20 pipes (Figure 7 ). This network is constrained to a maximum flow velocity of $3 \mathrm{~m} / \mathrm{s}$, minimum flow velocity of $0.6 \mathrm{~m} / \mathrm{s}$, maximum relative flow depth of 0.82 , minimum relative flow depth of 0.1 , and minimum cover depth of $2.45 \mathrm{~m}$. Constant

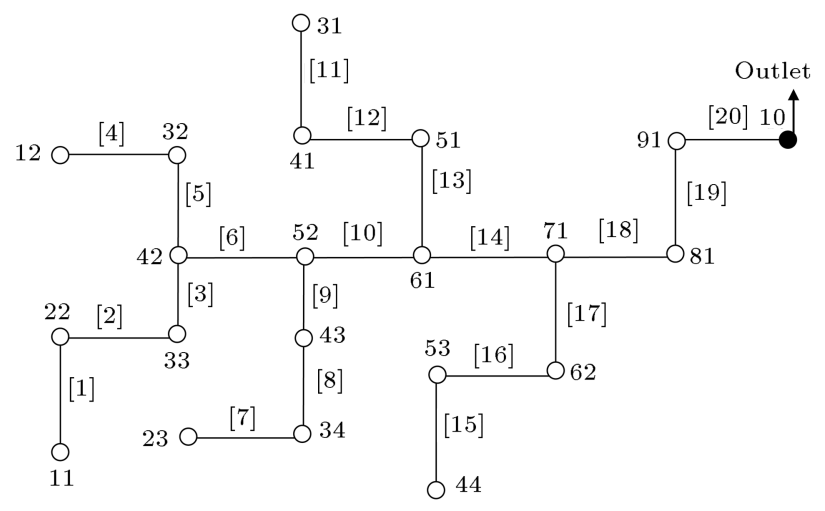

Figure 6. Test example I sewer network layout. 
Table 1. Data of test example I.

\begin{tabular}{|c|c|c|c|c|c|c|}
\hline \multirow[t]{2}{*}{$\begin{array}{c}\text { Pipe } \\
\text { no. }\end{array}$} & \multicolumn{2}{|c|}{$\begin{array}{c}\text { Node } \\
\text { no. }\end{array}$} & \multicolumn{2}{|c|}{$\begin{array}{c}\text { Ground } \\
\text { elevation } \\
(\mathrm{ft})\end{array}$} & \multirow[t]{2}{*}{$\begin{array}{c}\text { Length } \\
\text { (ft) }\end{array}$} & \multirow[t]{2}{*}{$\begin{array}{c}\text { Design } \\
\text { discharge } \\
\text { (cfs) }\end{array}$} \\
\hline & Up & Down & $\mathrm{Up}$ & Down & & \\
\hline 1 & 11 & 22 & 500 & 495 & 350 & 4 \\
\hline 2 & 22 & 33 & 495 & 487 & 400 & 3 \\
\hline 3 & 33 & 42 & 487 & 480 & 350 & 2 \\
\hline 4 & 12 & 32 & 490 & 485 & 400 & 4 \\
\hline 5 & 32 & 42 & 485 & 480 & 430 & 4 \\
\hline 6 & 42 & 52 & 480 & 470 & 550 & 5 \\
\hline 7 & 23 & 34 & 490 & 485 & 500 & 8 \\
\hline 8 & 34 & 43 & 485 & 475 & 450 & 4 \\
\hline 9 & 43 & 52 & 475 & 470 & 350 & 4 \\
\hline 10 & 52 & 61 & 470 & 465 & 500 & 6 \\
\hline 11 & 31 & 41 & 485 & 475 & 500 & 9 \\
\hline 12 & 41 & 51 & 475 & 470 & 350 & 7 \\
\hline 13 & 51 & 61 & 470 & 465 & 350 & 4 \\
\hline 14 & 61 & 71 & 465 & 455 & 565 & 7 \\
\hline 15 & 44 & 53 & 460 & 464 & 400 & 4 \\
\hline 16 & 53 & 62 & 464 & 460 & 300 & 2 \\
\hline 17 & 62 & 71 & 460 & 455 & 345 & 3 \\
\hline 18 & 71 & 81 & 455 & 451 & 400 & 7 \\
\hline 19 & 81 & 91 & 451 & 448 & 500 & 2 \\
\hline 20 & 91 & 10 & 448 & 445 & 612 & 5 \\
\hline
\end{tabular}

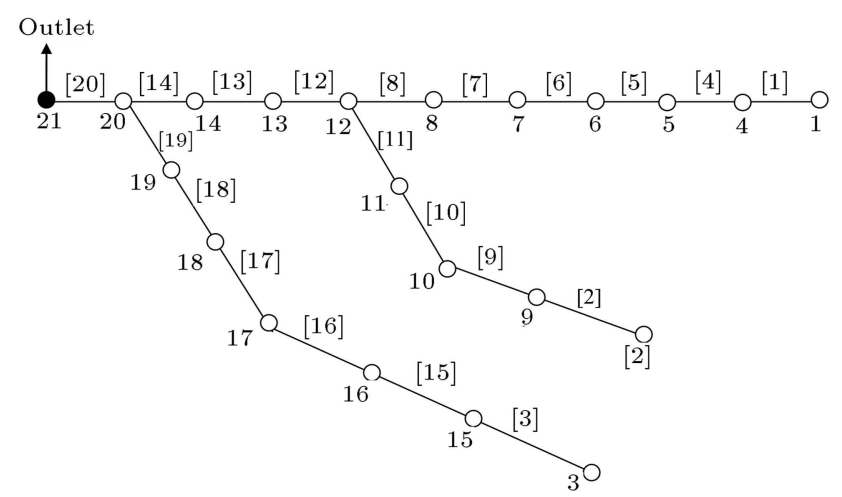

Figure 7. Test example II sewer network layout.

Manning coefficient is considered as 0.013. Details of the network, including nodal ground elevations and length and design discharge of each pipe, are given in Table 2. Commercial pipe diameters are 200, 250, 300, 400, 500, 600, and 700 millimetres, and other problem data of this test example are presented in the paper of Mansoury and Khanjani [44]. Here, the following relation is used for pipe installation and manhole costs:

$$
\begin{aligned}
& K_{\text {pip }}=1.93 e^{3.43} d_{l}+0.812 E_{l}+0.437 E_{l}^{1.53} d_{l}, \\
& K_{\text {man }}=41.46 h_{m} .
\end{aligned}
$$

\begin{tabular}{|c|c|c|c|c|c|c|}
\hline \multirow[t]{2}{*}{$\begin{array}{c}\text { Pipe } \\
\text { no. }\end{array}$} & \multicolumn{2}{|c|}{$\begin{array}{c}\text { Node } \\
\text { no. }\end{array}$} & \multicolumn{2}{|c|}{$\begin{array}{c}\text { Ground } \\
\text { elevation } \\
(\mathbf{m})\end{array}$} & \multirow[t]{2}{*}{$\begin{array}{c}\text { Length } \\
(\mathrm{m})\end{array}$} & \multirow[t]{2}{*}{$\begin{array}{c}\text { Design } \\
\text { discharge } \\
\left(\mathrm{m}^{3} / \mathrm{s}\right)\end{array}$} \\
\hline & $\mathbf{U p}$ & Down & $\mathbf{U p}$ & Down & & \\
\hline 1 & 1 & 4 & 74.59 & 73.66 & 260 & 27.9 \\
\hline 2 & 2 & 9 & 70.7 & 69.9 & 300 & 54.9 \\
\hline 3 & 3 & 15 & 73 & 71.50 & 400 & 21.1 \\
\hline 4 & 4 & 5 & 73.66 & 72.1 & 460 & 30.4 \\
\hline 5 & 5 & 6 & 72.1 & 71.99 & 260 & 32.4 \\
\hline 6 & 6 & 7 & 71.19 & 69.85 & 300 & 34 \\
\hline 7 & 7 & 8 & 69.85 & 68.24 & 450 & 36.6 \\
\hline 8 & 8 & 12 & 68.24 & 67.82 & 400 & 38.7 \\
\hline 9 & 9 & 10 & 69.9 & 69.3 & 270 & 56.2 \\
\hline 10 & 10 & 11 & 69.3 & 68.4 & 310 & 58 \\
\hline 11 & 11 & 12 & 68.4 & 67.28 & 440 & 59.6 \\
\hline 12 & 12 & 13 & 67.28 & 66.22 & 470 & 96.7 \\
\hline 13 & 13 & 14 & 66.22 & 65.82 & 350 & 101.2 \\
\hline 14 & 14 & 20 & 65.82 & 65.42 & 340 & 104.7 \\
\hline 15 & 15 & 16 & 71.5 & 70.1 & 400 & 26.4 \\
\hline 16 & 16 & 17 & 70.1 & 68.6 & 400 & 30 \\
\hline 17 & 17 & 18 & 68.6 & 66.8 & 500 & 31.9 \\
\hline 18 & 18 & 19 & 66.8 & 66.1 & 400 & 40.3 \\
\hline 19 & 19 & 20 & 66.1 & 65.42 & 590 & 44.6 \\
\hline 20 & 20 & 21 & 65.42 & 64.5 & 320 & 165.9 \\
\hline
\end{tabular}

Table 2. Data of test example II.

A set of preliminary runs is first done to find the proper values of MMAS parameters as shown in Table 3 for the proposed formulations. All the results presented hereafter are based on a uniform discretisation of the allowable range of cover depths into 30 intervals for all the proposed formulations and test examples. It should be noted that in PBACOA formulations, no heuristic information can be defined, and therefore, the value of $\beta=0.0$ is used.

The results of 10 runs carried out using different randomly generated initial guesses for the test examples along with the scaled standard deviation, the number of final feasible solutions, and the number of function evaluations and CPU time to get minimum solution cost are presented in Table 4 . It is clearly seen from Table 4 that all measures of the quality of the final solutions, such as the minimum, maximum and average costs, the number of final feasible solutions, and the numbers of function evaluations to get minimum solution cost, are improved when using ABACOA compared to PBACOA. Furthermore, it is seen that constrained versions of each PBACOA and ABACOA have been able to outperform the corresponding unconstrained version of these formulations regarding the quality of the solution due to the fact that the search 
Table 3. Values of MMAS parameters.

\begin{tabular}{ccccccccc}
\hline $\begin{array}{c}\text { Test } \\
\text { example }\end{array}$ & Formulation & Iteration & Ant & $\begin{array}{c}\text { Function } \\
\text { evaluation }\end{array}$ & $\boldsymbol{\alpha}$ & $\boldsymbol{\beta}$ & $\boldsymbol{\rho}$ & $\boldsymbol{p}_{\text {best }}$ \\
\hline \multirow{2}{*}{ I } & PBACOA & 1000 & 200 & 200000 & 1 & 0 & 0.95 & 0.2 \\
& ABACOA & 1000 & 200 & 200000 & 1 & 0.2 & 0.95 & 0.2 \\
\multirow{2}{*}{ II } & PBACOA & 1000 & 200 & 200000 & 1 & 0 & 0.95 & 0.2 \\
& ABACOA & 1000 & 200 & 200000 & 1 & 0.1 & 0.95 & 0.2 \\
\hline
\end{tabular}

Table 4. Maximum, minimum, and average solution cost values over 10 runs obtained with the proposed formulations.

\begin{tabular}{|c|c|c|c|c|c|c|c|c|}
\hline \multirow{2}{*}{ 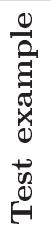 } & \multirow{2}{*}{ 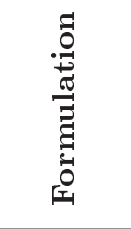 } & \multicolumn{3}{|c|}{ Solution cost value } & \multirow{2}{*}{$\begin{array}{c}\text { Scaled } \\
\text { standard } \\
\text { deviation }\end{array}$} & \multirow{2}{*}{$\begin{array}{c}\text { No. of runs } \\
\text { with final } \\
\text { feasible } \\
\text { solution }\end{array}$} & \multirow{2}{*}{$\begin{array}{l}\text { No. of function } \\
\text { evaluations to } \\
\text { get minimum } \\
\text { solution cost } \\
\text { value }\end{array}$} & \multirow{2}{*}{$\begin{array}{l}\text { CPU time } \\
\text { to get } \\
\text { minimum } \\
\text { solution cost } \\
\text { value (sec) }\end{array}$} \\
\hline & & Minimum & Maximum & Average & & & & \\
\hline \multirow{4}{*}{ I } & UPBAC & 237200 & 245751 & 240072 & 0.0106 & 10 & 158800 & 97 \\
\hline & $\mathrm{CPBAC}$ & 236287 & 239113 & 237757 & 0.0048 & 10 & 82400 & 58 \\
\hline & UABAC & 236287 & 242390 & 238497 & 0.0083 & 10 & 47200 & 100 \\
\hline & CABAC & 236287 & 237978 & 236658 & 0.0022 & 10 & 42800 & 63 \\
\hline \multirow{4}{*}{ II } & UPBAC & 78362.9 & 90725.7 & 82063.4 & 0.0561 & 10 & 147000 & 88 \\
\hline & CPBAC & 78190.4 & 79402.7 & 78780.7 & 0.0079 & 10 & 25600 & 14 \\
\hline & UABAC & 77674.1 & 90143.7 & 81191.2 & 0.0429 & 10 & 124000 & 98 \\
\hline & CABAC & 77674.1 & 77674.1 & 77674.1 & 0 & 10 & 15800 & 18 \\
\hline
\end{tabular}

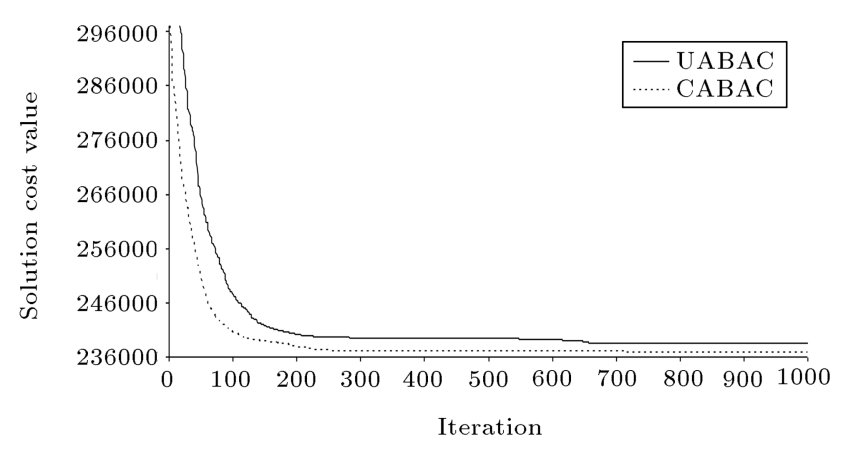

Figure 8. Variation of average solution cost values of test example I using unconstrained and constrained versions of ABACOA.

space size of the problem is much smaller than those of the unconstrained formulations.

Figures 8 and 9 show convergence curves of the average solution costs obtained in ten runs using unconstrained version of ABACOA compared to constrained version of it for test examples I and II, respectively. These figures indicate the superior performance of the constrained version of this formulation compared to unconstrained version of it in which the proposed constrained version leads to lower cost of the final solution during the evolution process. Furthermore, Figures 10 and 11 show convergence curves of the average solution costs obtained in ten runs using $\mathrm{CPBAC}$ and $\mathrm{CABAC}$ formulations for test examples

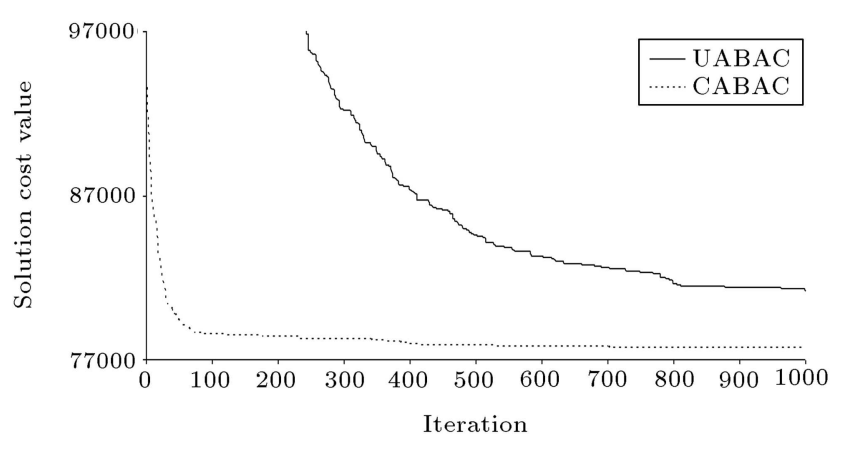

Figure 9. Variation of average solution cost values of test example II using unconstrained and constrained versions of $\mathrm{ABACOA}$.

I and II, respectively. These figures indicate superior performance of the CABAC compared to CPBAC. Finally, Figures 12 and 13 show the characteristics of the optimal solution obtained for test examples I and II, respectively, using CABAC in which the numbers in parentheses are nodal cover depths.

Test example I was solved by Mays and Wenzel using Differential Dynamic Programming (DDP) and the optimal solution of 265775 was reported [43]. Robinson and Labadie solved this problem later using DP, and the optimal solution of 275218 was reported [45]. This problem was later solved by Miles and Heaney using a spreadsheet template and 245874 was reported [46]. Furthermore, this problem was also solved by Afshar 


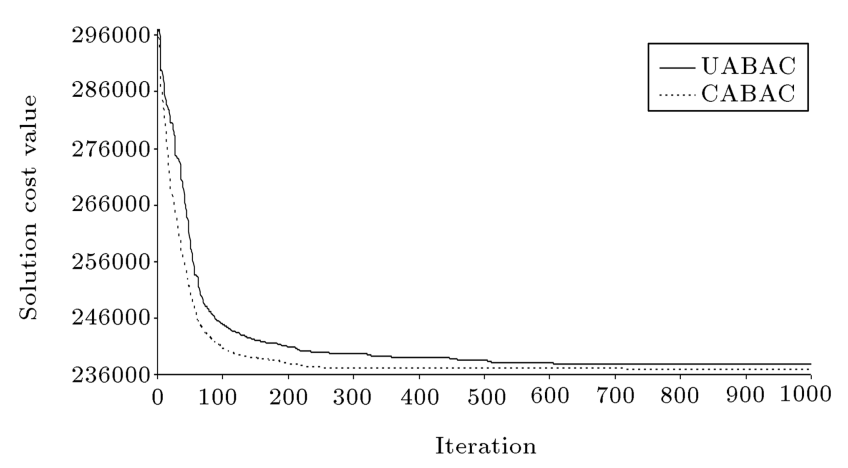

Figure 10. Variation of average solution cost values of test example I using CPBAC and CABAC.

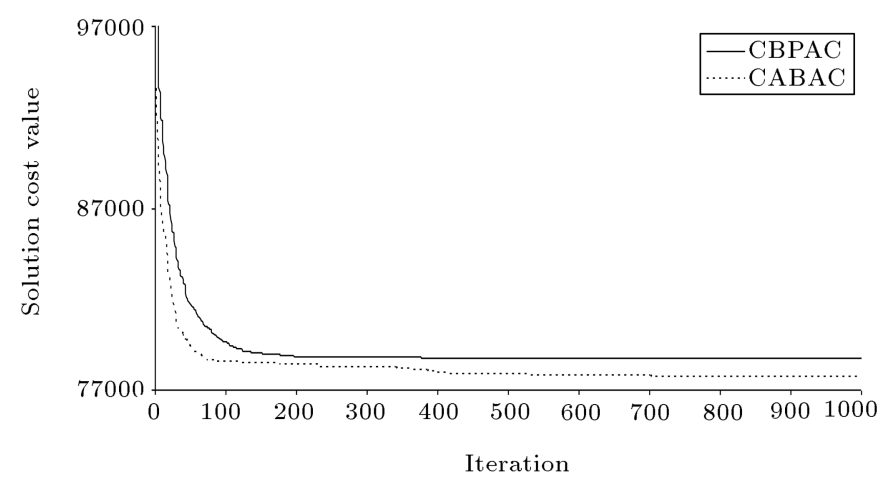

Figure 11. Variation of average solution cost values of test example II using CPBAC and CABAC.

proposing ACOA-AR (Adaptive Refinement process for ACOA), PCACOA, RPSO (Rebirthing Particle Swarm Optimization), and CACOA methods; the optimal solutions of 241496, 242539, 242162, and 242119 were respectively reported requiring 29900, 13900 , 30000 , and 20000 function evaluations [21,23,47,48]. In addition, Afshar et al. used Cellular Automata (CA) method in which it required 72 function evaluations to get the optimal solution of 253483 for this problem [24]. Later, Afshar and Rohani proposed discrete and continuous CA based hybrid methods to solve this problem, and the optimal solutions of 247412 and 248100 were reported requiring 69 and 7 function evaluations, respectively [35]. A GA-based model was proposed by Palumbo et al. to get the optimal solution of 251971 for this problem [26]. Finally, Yeh et al. used TS and SA to solve this problem, and the optimal solutions of 241770 and 244571 were respectively reported requiring 1034809 and 15932235 function evaluations [27]. These results can be compared with the cost value of 236287 obtained with 42800 function evaluations using the proposed CABAC for test example I, indicating the superiority of the proposed formulation with proper computational effort.

Test example II was solved, firstly, by Mansouri and Khanjani using GA and NLP, and the optimal solution of 83116 was reported [44]. NLP-BFGS, NLP-Fletcher-Reeves, and GA were used later by Setoodeh to solve this test example, and he reported the optimal solutions of 82732, 81553, and 77736, respectively [49]. Afshar et al. proposed CA method to solve this problem in which it required 15 function evaluations to get the optimal solution of 80879 [24]. Finally, this problem was solved by Afshar and Rohani who proposed discrete and continuous CA based hybrid methods, and the optimal solutions of 77327 and 77433 were reported requiring 45 and 38 function evaluations, respectively [35]. These results can be compared with the cost value of 77674.1 obtained with 15800 function evaluations using the proposed CABAC for this test example, indicating the superiority of the proposed formulation with proper computational effort.

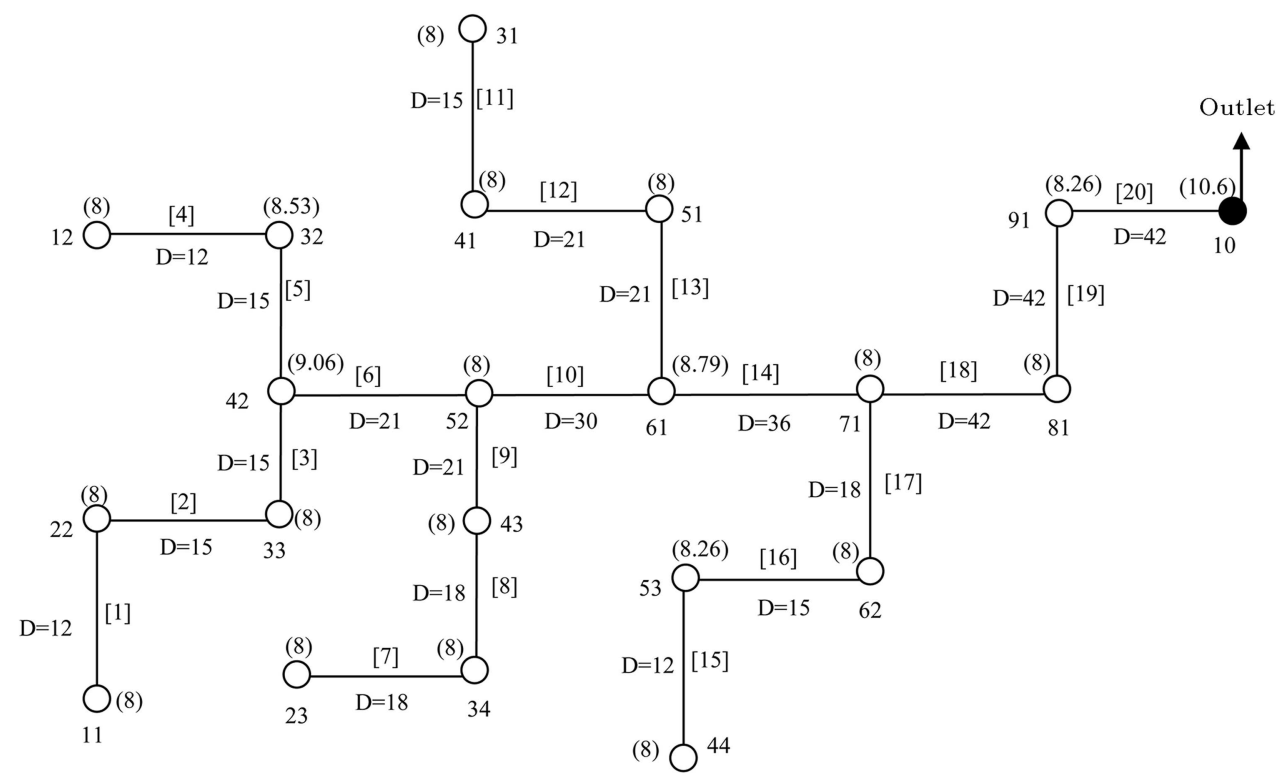

Figure 12. Characteristics of the optimal solution of example I using CABAC. 


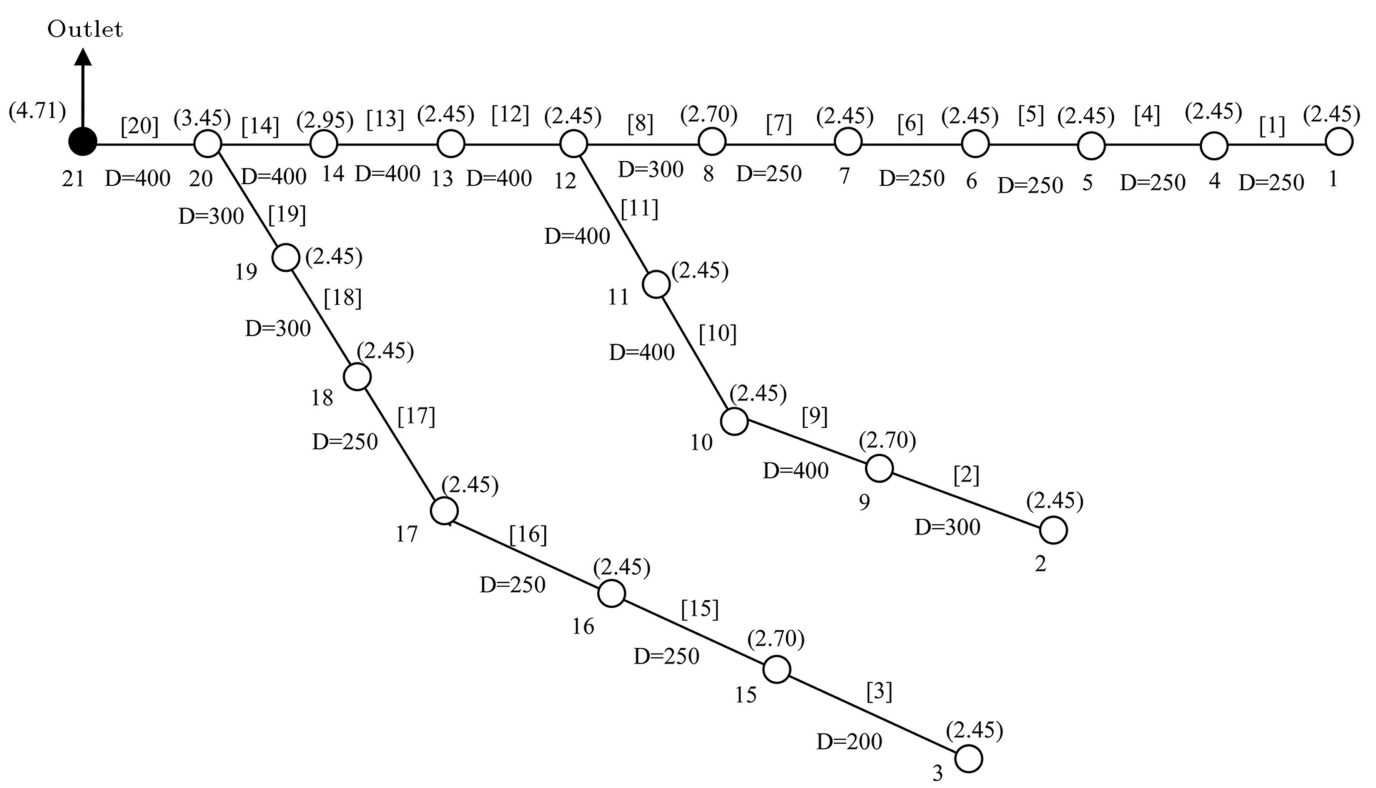

Figure 13. Characteristics of the optimal solution of test example II using CABAC.

\section{Conclusions}

Here, two different formulations of Arc Based Ant Colony Optimization Algorithm (ABACOA) were proposed to solve sewer network design optimization problem considering the cover depths of sewer network nodes as decision variables of the problem. Here, in the second formulation, the constrained version of ABACOA was proposed to satisfy slope constraint explicitly leading to reduction of search space of the problem. Two benchmark test examples were solved here using the proposed formulations, and the results were presented and compared with those obtained with alternative usual point-based formulation and other existing methods. Comparison of the results showed superiority of the proposed ABACOA to optimally solve the sewer network design optimization in which it has two distinct advantages of efficient implementation of the exploration and exploitation features and also an easy definition of the heuristic information for the ants over the alternative usual point-based formulation. Furthermore, the results indicate the ability of the constrained version of ABACOA to efficiently and effectively solve this problem in which it was shown to produce better results with smaller computational effort and less sensitivity to the randomly generated initial guess represented by the scaled standard deviation of the solutions produced in ten different runs in comparison with all other proposed formulations. In other words, the average solution costs of test examples I and II were improved $0.8 \%$ and $4.5 \%$, respectively, using CABAC in comparison with UABAC. In addition, the average solution costs of test examples I and II were improved $0.5 \%$ and $1.5 \%$, respectively, using arc based ACOA in comparison with point-based ones.
Finally, the obtained results of the proposed CABAC formulation for both of the test examples were better than all available results.

\section{References}

1. Moeini, R. and Afshar, M.H. "Sewer network design optimization problem using ant colony optimization algorithm and tree growing algorithm", In Proceeding of EVOLVE 2013, A Bridge Between Probability, Set Oriented Numerics, and Evolutionary Computation IV Advances in Intelligent Systems and Computing, Leiden, Netherlands, 227, pp. 91-105 (2013).

2. Haestad "Wastewater collection system modelling and design", Waterbury: Haestad Methods (2004).

3. Guo, Y., Walters, G. and Savic, D. "Optimal design of storm sewer networks: Past, Present and Future", In Proceeding of 11th International Conference on Urban Drainage, Edinburgh, Scotland, UK, pp. 1-10 (2008).

4. Desher, D.P. and Davis, P.K. "Designing sanitary sewers with microcomputers", Journal of Environmental Engineering, 112(6), pp. 993-1007 (1986).

5. Dajani, J.S. and Hasit, Y. "Capital cost minimization of drainage networks", ASCE Journal of Environmental Engineering, 100(2), pp. 325-337 (1974).

6. Elimam, A.A., Charalambous, C. and Ghobrial, F.H. "Optimum design of large sewer networks", Journal of Environmental Engineering, 115(6), pp. 118-89 (1989).

7. Swamee, P.K. and Shrama, A.K. "Optimal design of a sewer line using linear programming", Journal of Applied Mathematical Modelling, 37, pp. 4430-4439 (2013).

8. Price, R.K. "Design of storm water sewers for minimum construction cost", In Proceeding of 1 st International Conference on Urban Strom Drainage, Southampton, United Kingdom, pp. 636-647 (1978). 
9. Swamee, P.K. "Design of sewer line", Journal of Environmental Engineering, 127(9), pp. 776-781 (2001).

10. Walsh, S. and Brown, L.C. "Least cost method for sewer design", Journal of Environmental Engineering Division, 99(3), pp. 333-345 (1973).

11. Templeman, A.B. and Walters, G.A. "Optimal design of storm water drainage networks for roads", In Proceeding of Inst. of Civil Engineers, London, pp. 573-587 (1979).

12. Yen, B.C., Cheng, S.T., Jun, B.H., Voohees, M.L. and Wenzel, H.G. "Illinois least cost sewer system design model", User's Guide, Department of Civil Engineering, University of Texas at Austin (1984).

13. Botrous, A., El-Hattab, I. and Dahab, M. "Design of wastewater collection networks using dynamic programming optimization technique", In Proceeding of the ASCE Nat. Conf. on Environmental and Pipeline Engineering, Kansas City, MO, United States, American Society of Civil Engineers, pp. 503-512 (2000).

14. Diogo, A.F., Walters, G.A., Sousa, E.R. and Graveto, V.M. "Three-dimensional optimization of urban drainage systems", Computer Aided Civil Infrastructure Engineering, 15(6), pp. 409-426 (2000).

15. Gupta, A., Mehndiratta, S.L. and Khanna, P. "Gravity waste water collection systems optimization", $A S C E$ Journal of Environmental Engineering, 109(5), pp. 1195-1208 (1983).

16. Kulkarni, V.S. and Khanna, P. "Pumped wastewater collection systems optimization", ASCE Journal of Environmental Engineering, 111(5), pp. 589-601 (1985).

17. Heaney, J.P., Wright, L.T., Sample, D., Field, R. and Fan, C.Y. "Innovative methods for the optimization of gravity storm sewer design", In Proceedings the 8th International Conference on Urban Storm Drainage, Sydney, Australia, pp. 1896-903 (1999).

18. Liang, L.Y., Thompson, R.G. and Young, D.M. "Optimising the design of sewer networks using genetic algorithms and tabu search", Engineering, Construction and Architectural Management, 11(2), pp. 101112 (2004).

19. Guo, Y. "Sewer network optimal design based on cellular automata principles", In Proceeding of 2005 XXXI IAHR Congress, Seoul, Korea, pp. 6582-6593 (2005).

20. Afshar, M.H., Afshar, A., Marino, M.A. and Darbandi, A.A.S. "Hydrograph-based storm sewer design optimization by genetic algorithm", Canadian Journal Civil Engineering, 33(3), pp. 310-325 (2006).

21. Afshar, M.H. "Partially constrained ant colony optimization algorithm for the solution of constrained optimization problems: Application to storm water network design", Advances in Water Resources, 30(4), pp. 954-965 (2007).

22. Izquierdo, J., Montalvo, I., Perez, R. and Fuertes, V.S. "Design optimization of wastewater collection networks by PSO", Computers and Mathematics with Applications, 56(3), pp. 777-784 (2008).
23. Afshar, M.H. "A parameter free continuous ant colony optimization algorithm for the optimal design of storm sewer networks: Constrained and unconstrained approach", Advances in Engineering Software, 41, pp. 188-195 (2010).

24. Afshar, M.H., Shahidi, M., Rohania, M. and Sargolzaei, M. "Application of cellular automata to sewer network optimization problems", Scientia Iranica. Transactions A: Civil Engineering, 18(3), pp. 304-312 (2011).

25. Haghighi, A. and Bakhshipour, A.E. "Optimization of sewer networks using an adaptive genetic algorithm", Water Resource Management, 26(12), pp. 3441-3456 (2012).

26. Palumbo, A., Cimorelli, L., Covelli, C., Cozzolino, L., Mucherino, C. and Pianese, D. "Optimal design of urban drainage networks", Civil Engineering and Environmental Systems, 31(1), pp. 79-96 (2013).

27. Yeh, S.F., Chang, Y.J. and Lin, M.D. "Optimal design of sewer network by tabu search and simulated annealing", In Proceeding of International Conference on Industrial Engineering and Engineering Management (IEEM), 2013 IEEE, Bangkok, Thailand, pp. 16361640 (2013).

28. Cimorelli, L., Cozzolino, L., Covelli, C., Mucherino, C., Palumbo, A. and Pianese, D. "Optimal design of rural drainage networks", Journal of Irrigation and Drainage Engineering, 139(2), pp. 137-144 (2013).

29. Karovic, O. and Mays, L.W. "Sewer system design using simulated annealing in excel", Water Resource Management, 28, pp. 4551-4565 (2014).

30. Cimorelli, L., Cozzolino, L., Covelli, C., Della Morte, R. and Pianese, D. "Enhancing the efficiency of the automatic design of rural drainage network", Journal of Irrigation and Drainage Engineering, 140(6), pp. 040140151-0401401510 (2014).

31. Cozzolino, L., Cimorelli, L., Covelli, C., Mucherino, C. and Pianese, D. "An innovative approach for drainage network sizing”, Water, 7(2), pp. 546-567 (2015).

32. Cimorelli, L., Morlando, F., Cozzolino, L., Covelli, C., Della Morte, R. and Pianese, D. "Optimal positioning and sizing of detention tanks within urban drainage networks", Journal of Irrigation and Drainage Engineering, 142(1), pp. 040150281-0401502812 (2016).

33. Guo, Y., Walters, G.A., Khu, S.T. and Keedwell, E.C. "Optimal design of sewer networks using hybrid cellular automata and genetic algorithm", In Proceeding of IWA World Water Congress, Beijing, China (2006).

34. Pan, T.C. and Kao, J.J. "GA-QP model to optimize sewer system design", ASCE Journal of Environmental Engineering, 135(1), pp. 17-24 (2009).

35. Afshar, M.H. and Rohani, M. "Optimal design of sewer networks using cellular automata-based hybrid methods: Discrete and continuous approaches", Engineering Optimization, 44(1), pp. 1-22 (2012). 
36. Liu, C., Han, H., Wang, C. and Qiao, J. "An adaptive differential evolution algorithm for sewer networks design", In Proceeding of the 11th World Congress on Intelligent Control and Automation, Shenyang, China, pp. 3577-3583 (2014).

37. Rohani, M. and Afshar, M.H. "sewer networks optimization using cellular automata", Studies in Engineering and Technology, 1(1), pp. 1-12 (2014).

38. Rohani, M. and Afshar, M.H. "GA-GHCA model for the optimal design of pumped sewer networks", Canadian Journal of Civil Engineering, 42(1), pp. 1-12 (2015).

39. Moeini, R. and Afshar, M.H. "Application of an ant colony optimization algorithm for optimal operation of reservoirs: A comparative study of three proposed formulations", Scientia Iranica. Transactions A: Civil Engineering, 16(4), pp. 273-285 (2009).

40. Moeini, R. and Afshar, M.H. "Arc-based constrained ant colony optimization algorithms for the optimal solution of hydropower reservoir operation problems", Canadian Journal of Civil Engineering, 38, pp. 811824 (2011).

41. Afshar, M.H. and Moeini, R. "partially and fully constrained ant algorithms for the optimal solution of large scale reservoir operation problems", Journal Water Resource Management, 22(1), pp. 1835-1857 (2008).

42. Mays, L.W. and Yen. B.C. "Optimal cost design of branched sewer systems", Water Resources Research, 11(1), pp. 37-47 (1975).

43. Mays, L.W. and Wenzel, H.G. "Optimal design of multilevel branching sewer systems", Water Resour. Res., 12(5), pp. 913-917 (1976).

44. Mansouri, M.R. and Khanjani, M.J. "Optimization of sewer system by the nonlinear programming", $J$. of Water and Wastewater, 30, pp. 20-30 (1999) [In Persian].
45. Robinson, D.K. and Labadie, J.W. "Optimal design of urban storm water drainage system", Int. Symposium on Urban Hydrology, Hydraulics, and Sediment Control, University of Kentucky, Lexington, KY, USA, pp. 145-156 (1981).

46. Miles, S.W. and Heaney, J.P. "Better than optimal method for designing drainage systems", Journal of Water Resources Planning and Management, 114(5), pp. 477-499 (1988).

47. Afshar, M.H. "Improving the efficiency of ant algorithms using adaptive refinement: application to storm water network design", Advances in Water Resources, 29, pp. 1371-82 (2006).

48. Afshar, M.H. "Rebirthing particle swarm optimization algorithm: application to storm sewer network design", Canadian Journal of Civil Engineering, 35, pp. 11201127 (2008).

49. Setoodeh, M. "Optimal design of sewer networks", M.S. Thesis, Iran University of Science and Technology (2004).

\section{Biography}

Ramtin Moeini was born in Isfahan, Iran in 1981. He received his BS degree from Civil Engineering Department of Isfahan University of Technology (IUT) in 2002 and his MS degree (honors), and his PhD degrees (honors) from Civil Engineering Department of Iran University of Science and Technology (IUST) in 2007 and 2013, respectively. He has been an Assistant Professor of University of Isfahan since 2013. He is author/coauthor of 19 research articles in international and national journals and 20 conference papers. His research interests include optimization, evolutionary algorithms, water resource engineering, and water and sewer networks. 